\title{
TIBIAL FRACTURE IN AN ELDERLY FEMALE PATIENT OF RHEUMATOID ARTHRITIS: A PREVENTABLE IATROGENIC INJURY
}

\author{
Shambo Samrat Samajdar, Shatavisa Mukherjee*, Sumalya Sen, Santanu Kumar Tripathi \\ Department of Clinical and Experimental Pharmacology, \\ Calcutta School of Tropical Medicine, Kolkata, India.
}

\begin{abstract}
Oral systemic glucocorticoids widely used in the medical practice to treat various diseases like asthma, systemic connective tissue diseases, other autoimmune diseases and in transplantology, are considered the main cause of secondary and iatrogenic osteoporosis. The pathogenesis of glucocorticoid-induced bone loss is multifactorial and complex. The exact mechanism remains undefined. The present report describes a case of 65 years old female presenting with fracture in mid shaft of tibia after a very low velocity trauma. On medical history elicitation, she was found to be treated for rheumatoid arthritis with oral glucocorticoids. Causality, severity and preventability assessment was done for the reaction. Potentiality of glucocorticoids causing fractures is often neglected by professionals and ignored by patient and their caregivers. Given the potential for rapid bone loss with glucocorticoid therapy, frequent monitoring is warranted while bearing in mind that BMD is a surrogate marker for fracture risk and patients on glucocorticoids fracture at higher BMD than other patients. Once treatment is initiated, BMD should be monitored annually to ensure compliance and efficacy.
\end{abstract}

Keywords: Glucocorticoids, Bone loss, Osteoporosis, Bone fracture, Bone mineral density.

\section{Introduction}

Oral systemic glucocorticoids (GC) are widely used in medical practicesin transplantology and for treating asthma, systemic connective tissue disorders, and various autoimmune diseases. The therapy is usually continued for more than three months. However, on chronic use, GCs are considered one of the contributing causes of secondary and iatrogenic osteoporosis. Studies in various centres have shown that usage of $5 \mathrm{mg}$ predinisone per day causes increased bone loss in the initial 6 to 12 months with low daily dosing of $2.5 \mathrm{mg}$ of prednisolone, in intermittent and repeated cycles, also having a cumulative and deleterious effect.[1] The risk is usually higher in the initial three months of continuous therapy and slowly decreases after its discontinuation; however, it does not resume normalcy. [2]

The GC-induced bone disease mainly affects trabecular bone, justifying the higher occurrence of vertebral and rib fractures. The potentiality of GC causing fractures is often neglected by professionals and ignored by the patient and their caregivers. Relative risk increases with dose and duration of therapy, while the absolute risk can be determined by a range of associated clinical conditions. The present report describes a case of GC induced fracture in an elderly female patient of rheumatoid arthritis.

eISSN: 2523-6709

pISSN: 2523-6695

DOI: $10.31878 /$ ijcrpp.2019.33.2

\section{Case Description}

A 65 years old normotensive euglycemic female presented with a fracture in mid shaft of tibia following a very low-velocity trauma. On history elicitation, she was found to have been treated for rheumatoid arthritis with methylprednisolone $4 \mathrm{mg}$ once daily, sulfasalazine $500 \mathrm{mg}$ twice daily and vitamin D3 calcium supplementation for last one year. The patient had a sedentary lifestyle and a positive familial history of osteoporotic fracture. She was advised to continue all medicines with the addition of alendronic acid $70 \mathrm{mg}$ once weekly with plaster cast being done.

After four weeks, the patient presented with a burning sensation in the throat, along with severe hyperacidity. Alendronate was withdrawn. Pantoprazole and domperidone combination was prescribed. Laboratory investigations suggested FPG $98 \mathrm{mg} / \mathrm{dl}$, serum creatinine - $0.8 \mathrm{mg} / \mathrm{dl}$, hemoglobin - $11.7 \mathrm{gm} / \mathrm{dl}$, serum calcium - $9.80 \mathrm{mg} / \mathrm{dl}$, alkaline phosphatase- $100 \mathrm{U} / \mathrm{L}$, $\mathrm{TSH}$ - $3.1 \mathrm{uIU} / \mathrm{ml}$, Vit $25 \mathrm{OH} \mathrm{D}$ - $40 \mathrm{ng} / \mathrm{ml}$, normal liver profile. Dual-energy X-ray absorptiometry (DEXA) scan revealed T-score of -3.1 at the femoral neck and T-score of - 2.9 at spine. FRAX score was 23. The patient was advised injection teriparatide $20 \mathrm{mcg}$ SC once daily, methotrexate $15 \mathrm{mg}$ once weekly, folic acid $5 \mathrm{mg}$ four days in a week, hydroxychloroquine $200 \mathrm{mg}$ twice daily, sulfasalazine $1000 \mathrm{mg}$ twice daily, calcium and vitamin D supplementation. On review after two months, patients presented with no more complaints, methotrexate dose was down titrated to $7.5 \mathrm{mg}$ once weekly with other medications continuing.

Correspondence: Ms. Shatavisa Mukherjee, Department of Clinical and Experimental Pharmacology, Calcutta School of Tropical Medicine, Kolkata, E-Mail: shatavisa100@gmail.com 
Causality assessment of this adverse event was found to be 'Possible' under WHO UMC Causality Assessment Scale.[3] A score of 3 could be attributed under Naranjo's algorithm[4], which confers it to be 'Possible'. The event was 'preventable' under Schumock and Thornton's preventability assessment criteria [5]. Severity assessment using Hartwig Seigel's Scale [6] conferred it to be moderate (Level 3). The event was reported under the National Pharmacovigilance Programme.

\section{Discussion}

Bone and muscle form a unit, with mutual trophic influence. Chronic GC use causes decreased bone remodelling and low bone turnover. Trabecular bone loss, including vertebrae and the femoral neck, are usually the most significantly affected ones.[7]Fracture risk at a specific bone mineral density (BMD) is greater for patients on GC therapy than the same BMD in a non -GC-treated patient, making it imperative that clinicians use different BMD thresholds for initiating osteoporosis treatments in these patients. Studies have shown a strong association between oral GC dose and fracture risk with greatest risk being during the initial 3-6 months of treatment initiation with GC.

Pathogenesis of GC induced bone loss is intricate. GCs increase the expression of parathyroid hormone (PTH) receptors in osteoblasts and enhance osteoblasts response to PTH. GC inhibits intestinal calcium absorption by vitamin $\mathrm{D}$ independent factors. Expression of the gene encoding calbindin-D28K, a protein involved in intestinal calcium transport is also implicated. In patients receiving GCs, serum levels of vit. D metabolites are usually in the normal range, serum levels of PTH are variable and generally has been reported to be normal. GCs produces a dose-related inhibition of osteoblasts maturation precursors, with a decreased number of osteoblasts in the matrix and the synthesis of both type I collagen and non-collagenous proteins by osteoblasts. GC inhibits gonadotropins, sex steroids and adrenal androgen production and release, which accelerates bone loss. Inhibition of prostaglandin E2, transforming growth factor $\beta 1$ and transcription and synthesis of Insulin-like growth factors are also implicated, which are a direct or indirect determinant of bone health.

GCs also have indirect effects on local growth factors such as fibroblast growth factors, platelet-derived growth factors, bone morphogenetic proteins, cytokines like IL 6, 11, colony stimulating factors. With direct action on the collagen and collagenases, GC causes the decreased bone formation and increased resorption. In the initial phase of therapy, there is increased osteoclastic activity coupled with increased bone resorption leading to rapid loss of bone integrity and significant fracture risk. Later in the course of treatment, resorption slows, resulting in a state of chronically decreased bone turnover. Osteoblast signaling is also affected, causing reduced osteoprotegerin release and increased receptor activator of NF-KB ligand, resulting in osteoclastogenesis. Other actions of $\mathrm{GC}$ that negatively affect bone include decreased calcium absorption by the gastrointestinal tract and renal calcium loss. Fracture risk is also associated with steroid myopathy resulting in muscle weakness. $[8,9]$
The GC-induced bone loss remains an important consequence of GC therapy, which is partially reversible, and hence, prevention should be considered and discussed in all patients during initiation of GC treatment. Patients receiving GC on a long-term basis should have a calcium intake of $1,500 \mathrm{mg} / \mathrm{d}$, and their hypercalciuria should be controlled by sodium restriction, and if necessary, a thiazide combined with a potassium-sparing diuretic. Gonadal hormones should be replaced when deficient, and adequate levels of 25 $\mathrm{OH}$-vitamin D should be assured. Physical activity should be encouraged. Preliminary studies suggest that bisphosphonates may be useful in the prevention of bone loss. Calcitonin and sodium fluoride may be useful in patients with significant GC-induced osteoporosis (GIO). It is time to be aggressive in preventing and treating GC-induced bone loss in all patients regardless of symptoms. For premenopausal women and men under the age of 50 years, fracture risk should be determined, and treatment decisions based on this assessment. In postmenopausal women and older men, the risk of GIO and fractures is greater and the evidence demonstrating the benefit of preventative therapy is clear. In our opinion, these patients should be initiated on preventative therapy. Currently, guidelines recommend oral bisphosphonate therapy as first-line treatment, with adjuvant calcium and vitamin D. New evidence suggests greater BMD gains with zoledronic acid and PTH and these therapies may, therefore, provide important options particularly in high-risk patients with established GIO. [10]

\section{Conclusion}

Given the potential for rapid bone loss with GC therapy, frequent monitoring is warranted while bearing in mind that $\mathrm{BMD}$ is a surrogate marker for fracture risk and patients on GC fracture at higher BMD than other patients. Once treatment is initiated, BMD should be monitored annually to ensure compliance and efficacy. Further research is needed to define in an evidencebased manner the most efficient frequency of BMD monitoring and the optimal duration of treatment with bisphosphonates.

\section{Source of Support: Nil}

\section{Conflict of Interest: None Declared}

Acknowledgement: The authors acknowledge and support the untiring efforts and contribution of Pharmacovigilance Programme of India (PvPI) towards ensuring better patient safety nationwide.

\section{References}

[1] Amiche MA, Abtahi S, Driessen JHM, et al. Impact of cumulative exposure to high-dose oral glucocorticoids on fracture risk in Denmark: a population-based case-control study. Arch Osteoporos. 2018;13(1):30.

[2] Fraser LA, Adachi JD. Glucocorticoid-induced osteoporosis: treatment update and review. Ther Adv Musculoskelet Dis. 2009;1(2):71-85.

[3] The use of the WHO-UMC system for standardised case causality assessment. Accessed from http://who-umc.org/Graphics/24734.pdf. 
Accessed on Jun 03, 2017.

[4] Naranjo CA, Busto U, Sellers EM. A method for estimating the probability of adverse drug reactions. ClinPharmacolTher. 1981; 30(2):23945 .

[5] Schumock GT, Thornton JP. Focusing on the preventability of adverse drug reactions. Hosp Pharm. 1992;27(6):538.

[6] Hartwig SC, Siegel J, Schneider PJ. Preventability and severity assessment in reporting adverse drug reactions. Am J Hosp Pharm. 1992;49(9):2229-32

[7] Hunter DJ, Sambrook PN. Bone loss. Epidemiology of bone loss. Arthritis Res. 2000;2 (6):441-5.

[8] Sarinho ESC, Melo VMPP. Glucocorticoidinduced bone disease: mechanisms and importance in pediatric practicedoençaósseainduzidapelosglicocorticoide s: mecanismos e importâncianapráticapediátrica. Rev Paul Pediatr. 2017;35(2):207-15

[9] McIlwain HH. Glucocorticoid-induced osteoporosis: pathogenesis, diagnosis, and management. Prev Med. 2003;36(2):243-9.

[10] Bijlsma JW. Prevention of glucocorticoid induced osteoporosis. Ann Rheum Dis. 1997;56 (9):507-9. 